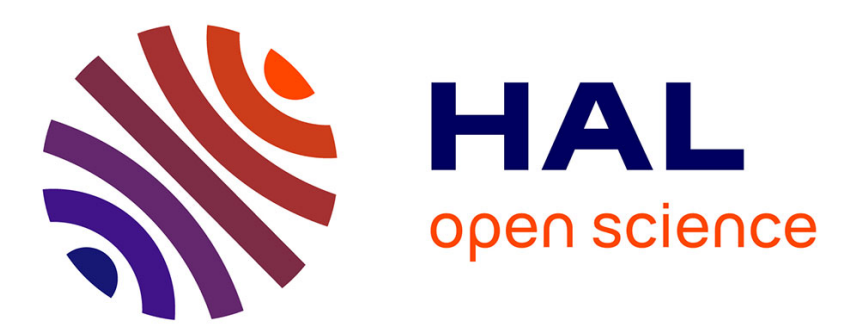

\title{
A vibration-free, thermally controlled setup for mechanical thermal noise measurements
}

S. Longo, L. Cecchinato, M. Rampazzo, M. Bonaldi, A. Beghi, L. Conti

\section{To cite this version:}

S. Longo, L. Cecchinato, M. Rampazzo, M. Bonaldi, A. Beghi, et al.. A vibration-free, thermally controlled setup for mechanical thermal noise measurements. European Physical Journal: Applied Physics, 2012, 57 (2), 10.1051/epjap/2011110304 . hal-00768703

\section{HAL Id: hal-00768703 https://hal.science/hal-00768703}

Submitted on 23 Dec 2012

HAL is a multi-disciplinary open access archive for the deposit and dissemination of scientific research documents, whether they are published or not. The documents may come from teaching and research institutions in France or abroad, or from public or private research centers.
L'archive ouverte pluridisciplinaire HAL, est destinée au dépôt et à la diffusion de documents scientifiques de niveau recherche, publiés ou non, émanant des établissements d'enseignement et de recherche français ou étrangers, des laboratoires publics ou privés. 


\title{
A vibration-free, thermally controlled setup for mechanical thermal noise measurements
}

Stefano Longo $^{1}$, Luca Cecchinato ${ }^{2}$, Mirco Rampazzo $^{3}$, Michele Bonaldi $^{4,5}$, Alessandro Beghi ${ }^{3}$, and Livia Conti ${ }^{1}$ a

1 INFN, Sezione di Padova, Via Marzolo 8, I-35131 Padova, Italy

2 Dipartimento di Fisica Tecnica, Università degli Studi di Padova, via Venezia 1, I-35131 Padova, Italy

3 Dipartimento di Ingegneria dell'Informazione, Università degli Studi di Padova, Via Gradenigo 6/B, I-35131, Padova, Italy

${ }^{4}$ Istituto di Fotonica e Nanotecnologie, CNR-Fondazione Bruno Kessler, 38100 Povo, Trento, Italy

5 INFN, Gruppo Collegato di Trento, Sezione di Padova, 38100 Povo, Trento, Italy

Received: date / Revised version: date

\begin{abstract}
We have developed a setup that offers a vacuum compatible, vibration-free experimental space with active thermal stabilization around room temperature, suitable for high sensitivity measurements. In this paper we describe the experimental setup from the thermal viewpoint and the design and implementation of the thermal control. Then we characterize the long-term performances of the control loop by using experimental data collected over 1 month: we show that our control is effective in reducing room temperature variations by a factor 100 over a few weeks, even in presence of an internal dc heat source of $2 \mathrm{~W}$.
\end{abstract}

\section{Introduction}

To carry out a precise/high sensitivity physics measurement it is often required to operate in a very quiet and thermally controlled environment. Often this means: to house the apparatus in vacuum; to reduce mechanical vibrations transmitted by the pavement; to stabilize actively the temperature while minimizing touching the appara-

a Corresponding author: lconti@pd.infn.it tus. For instance, to measure the thermal noise induced vibration fluctuations of kg-scale, low-loss mechanical oscillators resonating at $1-2 \mathrm{kHz}$, we need to provide: (i) a reduction by at least a factor $10^{5}$ of mechanical noise at the oscillator input and around their working frequency and (ii) a reduction of the temperature changes of the oscillators down to a level which depends on both loss magnitude and measurement time scale. The first requirement is determined by the need to suppress ambient noise 
and the second requirement comes from the need to perform measurements at constant oscillator characteristics and noise.

Electronic temperature controllers have been described by numerous authors in the past mainly with focus on achieving a very high temperature stability in the system being controlled either at room or cryogenic temperature [1]. At the same time, there has been a lot of work on the development of mechanical suspensions for different payload masses and working frequencies [2]. However to our knowledge there is no description for a complete and versatile setup that addresses both the issues of vibration reduction and thermal control. We also note that these two features tends to contrast each other: the thermal control requires good thermal coupling between the controlled system and the thermal actuator, usually satisfied by using large cross-sections pieces, while the vibration reduction requires the system to have minimal mechanical coupling with the outer environment.

Our scientific goal is to measure the vibration fluctuations of low-loss mechanical oscillators when subjected to non-equilibrium steady-states due to thermal gradients [3]. We have already described the mechanical setup we developed to provide our oscillators with the required suspension in a vacuum environment [4] so to measure their mechanical thermal noise. In this paper we describe the thermal control of the payload: main requirements are not to spoil the mechanical attenuation provided by the suspension, to control the oscillator temperature within $0.1 \mathrm{~K}$ and to be able to setup a constant temperature difference across the oscillator of $\lesssim 15 \mathrm{~K}$ while not touching the oscillator itself. Since to fulfill our goal we need to populate the low-probability tails of the displacement noise statistical distribution, we need to run our experiment continuously for long periods (weeks): thus the above figures should be maintained for correspondingly long periods.

Our design can be easily reproduced whenever a quiet environment (for what regards both vibration and temperature) with a volume of a few (eg 1-3) liters is needed. As an added benefit, thanks to the simplicity of our mechanical design, we were able to develop the thermal model using the simple thermal-electrical analog thus avoiding to use commercial, finite elements simulation software.

In this paper we describe the design and implementation of the thermal control on the basis of the mechanical design described in ref. [4]. We also describe the experimental measurements we made to characterize the performance of the thermal control.

\section{The experimental setup}

The mechanical setup was mainly described in ref. [4] (see also fig. 1). It consists of an aluminum, cylindrical vacuum chamber on top of a commercial research grade optical table. The table legs constitute a low frequency stage of mechanical suspension, being themselves pneumatic isolators. The vacuum chamber is pumped by a vibration-free ion pump and hosts a 3 stage mechanical suspension. The stages are mounted one on top of the other: hence, at the final top stage the mechanical noise is minimum. Each sus- 
pension stage consists of $3 \mathrm{C}$-shaped, Al7075 - T6 springs connected in parallel to a single, annular stainless steel (AISI 304) load mass: the load masses of the two bottom single stages are identical and we named them shape A load masses. The load mass of the top stage is different from the lower two since it must support the payload: we named it shape B load mass. The payload consists of an Al5056 flange which houses the amplifier of the oscillator capacitive readout and the oscillator, bolted to the flange.

The oscillator occupies the free space left by the central hole of the annular masses (see fig. 1). The three stages of the suspension are assembled together using $\mathrm{Cu}$ threaded rods. The setup provides for a mechanical suspension able to reduce by $180 \mathrm{~dB}$ the mechanical noise at the oscillator input at about $1.5 \mathrm{kHz}$ while being effective in the frequency range $0.2-2 \mathrm{kHz}$. The same setup can be used to suspend with similar performance any payload of a few liters in volume, up to $\sim 7 \mathrm{~kg}$ in mass.

Fig. 2 and fig. 3 show respectively the 3-dimensional drawing of our oscillator assembly and one photograph of it. The oscillator consists schematically in a rod which is kept in the vertical position and whose top end is fixed, being anchored to the top annular steel mass; the rod bottom end is loaded with a mass $(\sim 0.2 \mathrm{~kg})$ and is free to vibrate. The oscillator is made of commercial aluminum alloy (namely Al6082) except for the sample (vertical darker element in the photograph) which is a single-crystal silicon rod. The rod is $5 \mathrm{~mm}$ in diameter and $100 \mathrm{~mm}$ in total length: it is glued at both ends (we used Stycast FT2850 with hardener $24 \mathrm{LV}$ ) to the aluminum pieces and

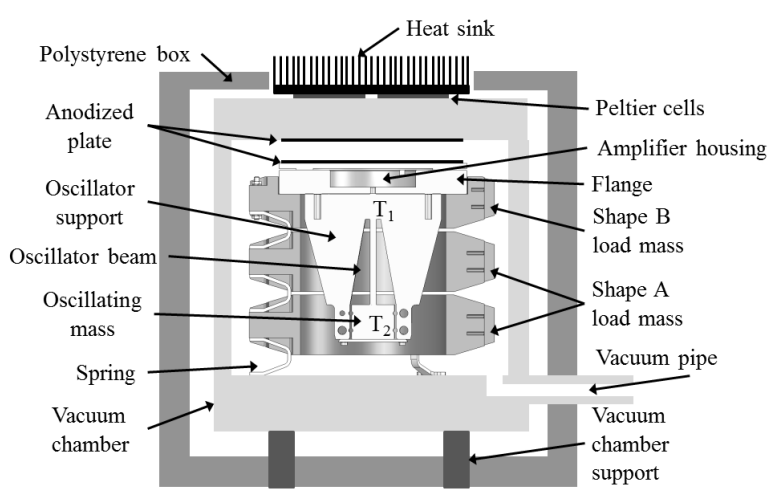

Fig. 1. Drawing (not to scale) of the cross-section of the experimental apparatus with indication of the components involved in the thermal control. Location of thermometers sensing oscillator base temperature $T_{1}$ and oscillator end mass temperature $T_{2}$ are also shown and labeled as their corresponding temperature.

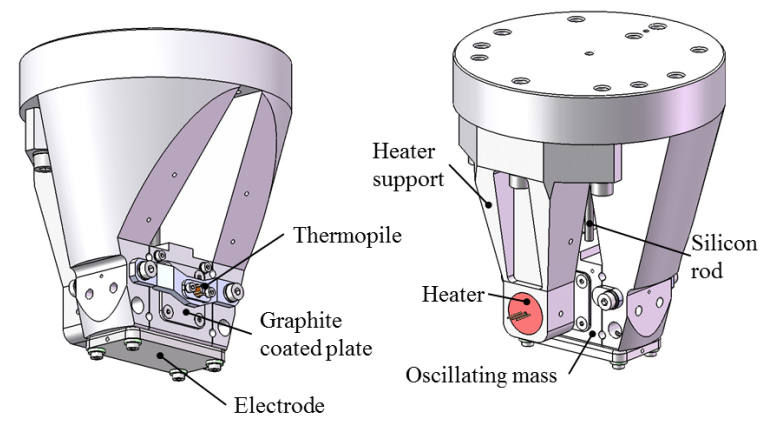

Fig. 2. Two views of the 3-dimensional drawing of the oscillator assembly with indications of main parts.

the emerging part is $85 \mathrm{~mm}$ in length. The bottom surface of the oscillating mass is faced to an aluminum plate (electrode, see fig. 2) to realize a capacitive sensing of the mass oscillations. The electrode is supported by two arms at the sides of the mass and it is electrically insulated from them by a $50 \mu \mathrm{m}$ thick teflon spacer (see fig. 3) which realizes the capacitor gap. A similar oscillator assembly, but with an aluminum rod, is described in ref. [3]. 


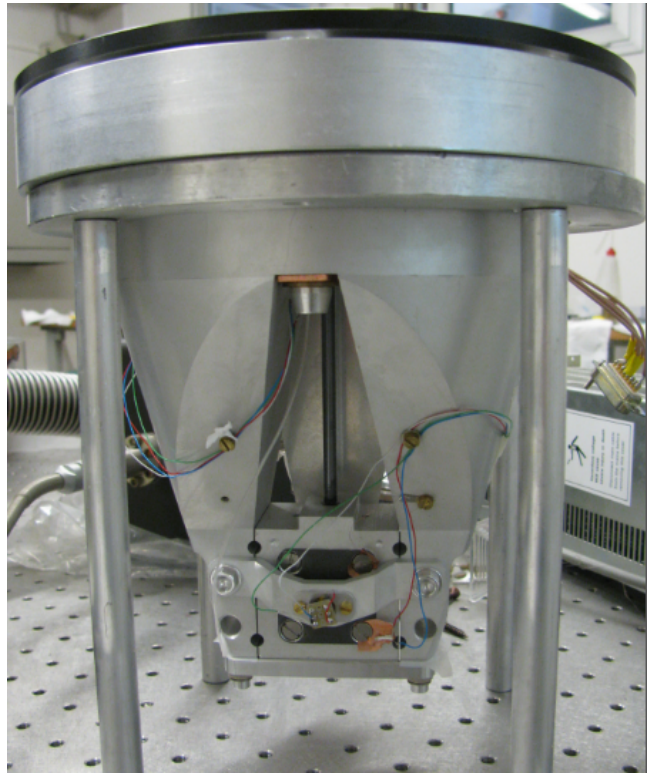

Fig. 3. Photograph of the oscillator assembly: the oscillator is supported by a frame resting on 4 legs. The vertical, silicon rod glued at top and bottom end is shown. One of the two opposite graphite coated areas is also shown. It is faced by the thermopile supported by a metal bridge connecting the electrode supports. At a corner of the coated area the Pt100 thermometer is visible, glued to a copper strip. At the rod top the copper plate can be seen that supports the NTC thermistor for $T_{1}$ measurements. The top, black plate in the photograph is the anodized plate that comes at the top of the suspension and which realizes the radiative coupling to the vacuum box top. The flat plate under the oscillating mass is the capacitor electrode: in the picture the $50 \mu \mathrm{m}$ thick teflon spacers can also be appreciated.

Once able to perform the (equilibrium) thermal noise measurements, as stated, our main goal [3] is to measure the oscillator vibrations in non-equilibrium steady states due to a temperature difference $\Delta T=T_{2}-T_{1}$ across the oscillator. We indicate $T_{1}$ the temperature of the oscillator base (ie at the top of the vertical rod) and $T_{2}$ the temperature of the oscillating mass (ie at the bottom end of the Silicon rod), as shown in fig. 1. Thus, as part of the non-equilibrium measurement, we had to develop a thermal control so to: 1) stabilize the temperature $T_{1}$ within $0.1 \mathrm{~K}$ for weeks and 2) apply a controlled temperature difference $\left(T_{2}-T_{1}\right.$ order of $\left.15 \mathrm{~K}\right)$ across the oscillator. Given our oscillator figures and the silicon thermal conductivity (see also tab. 1 and tab. 2), to set a temperature difference of $15 \mathrm{~K}$ we need to put about $0.5 \mathrm{~W}$ through the rod. The above two requirements need to be satisfied without touching the oscillator nor spoiling the mechanical suspension nor introducing noise.

To complete both tasks while working around room temperature, we decided to control $T_{1}$ by radiative cooling and $T_{2}$ by radiative heating. Fig. 1 provides insight on our thermal control strategy: we place Peltier cells on top of the vacuum box, in air, and cool radiatively the oscillator base. Thus we add a radiative heat exchange in parallel to the heat conduction through the vacuum box and suspension. To improve radiative heat exchange between the vacuum box top and the top of the suspension (both realized in aluminum) we subjected the facing surfaces to hard anodization [5]: thus their emissivity increases to more than 0.9 (for $\lambda>6 \mu \mathrm{m})$ from about 0.2 for oxidized aluminum. A similar strategy we adopted to heat one end of the aluminum oscillator by facing it to an infrared heater. Since we need to guarantee good coupling even below $6 \mu \mathrm{m}$ to cover the emission spectrum of the source, in this case we used a different surface treatment 
and sprayed a graphite black-coating $[6,7]$ on the portion of the oscillator surface facing the source.

To shield against ambient temperature changes we placed the whole setup in a box made of a passive insulator, namely Polystyrene (see fig. 1). The heat sink on top of the Peltier cells, the vacuum pipe and the 3 glass fibre reinforced plastic spacers that support the vacuum chamber are the only parts emerging from the Polystyrene box.

\subsection{Instrumentation}

To realize the thermal stabilization and to be able to add a temperature difference across the oscillator rod, we made use of different types of sensors and actuators. Wherever there was no major concern of adding mechanical losses, we used NTC thermistors to measure the temperature: this choice is justified by their large availability, low cost, small size, high sensitivity and even good long-term stability. In our application the latter feature is particularly important for the case of the oscillator base and mass temperatures, $T_{1}$ and $T_{2}$, since our experiment needs to run for long times (order of several weeks) with constant temperature difference around a constant temperature. Thus to measure $T_{1}$ we use a miniature bead-in-glass thermistor with dimension order of $1.5 \mathrm{~mm}$; the quoted long-term stability is a maximum $0.02 \%$ change per year in the resistance value at $105^{\circ} \mathrm{C}$. The resistance value at $25^{\circ} \mathrm{C}$ is about $2.5 \mathrm{k} \Omega$. The thermistor is glued to a rectangular copper plate fixed close to the top end of the oscillator rod (see also fig.3). Since the length of wires necessary to connect the probe to the measuring devices is a couple of meters, and in order to avoid errors due to wire resistance changes, we adopted a 4-wire configuration strategy to measure of $T_{1}$. Less performing thermistors and a simpler 2-wire scheme are adopted to measure the temperature of the room hosting the apparatus (which we call 'laboratory temperature' in the following), the temperature of the vacuum box top and the temperature of the heat sink.

To measure the temperature $T_{2}$ of the oscillating mass we adopted a non contact device, namely a thermopile (model A2TPMI 334-L5.5 OAA060 by PerkinElmer): the sensor incorporates a pre-amplification and ambient temperature compensation in a compact TO-39 housings (diameter: $9.3 \mathrm{~mm} \max )$. It provides for two calibrated voltage output: one is the object temperature, ie the temperature of the facing surface, ie $T_{2}$ in our case, and the other is the sensor case temperature. During the experimental run reported in this paper we did not acquire the thermopile signal measuring the case temperature. The sensor posses an integrated Si lens, $5.5 \mathrm{~mm}$ focal length and the field of view is $7^{\circ}$; it is supported by an aluminum bridge that is fixed to the supports of the electrode, at the sides of the oscillating mass (see also fig. 3). Given a distance of about $2 \mathrm{~mm}$ between the sensor and the facing surface of the oscillating mass, the sensed area is about $0,05 \mathrm{~mm}^{2}$. In order to reduce the contribution to the measurement coming from radiation emitted from other surfaces, we treated the sensed surface of the oscillating mass with the same graphite spray as for the area facing the IR heater: thus the contribution of the sensed area is increased by a factor 
equal to the emissivity ratio of the graphite vs aluminum, which is about a factor 10 .

For the experimental run reported in this paper we also fixed a miniature (size approx $2 \mathrm{~mm}$ ), platinum resistance thermometer Pt100 on the oscillating mass: we glued it to a copper strip which then we screwed to the mass. This was done to check if the thermopile was working properly because we did not find reports about its operation at low pressures. The thermopile is manufactory sealed in a dry nitrogen atmosphere. Thus, for the data reported in this paper we have two independent measurement of the oscillator mass: $T_{2, T}$ provided by the thermopile and $T_{2, P t}$ given by the Pt100.

To heat the oscillating mass and thus increase $T_{2}$ we use a non-coherent, thermal source (model IR-12K manufactured by Scitec Instruments) which works by heating electrically an element so that it emits in the infrared range. The heater faces the oscillating mass being fixed to an aluminum support attached to the oscillator base (see also fig. 2). The active area of the source is $3.5 \mathrm{~mm} \times 3.5 \mathrm{~mm}$ and the emissivity is 0.8 : thus the spectral radiance output is ( $80 \%$ of) that of a black body at a temperature depending on the fed current, in the $1 \mu \mathrm{m}$ to $22 \mu \mathrm{m}$ range. From an electrical view point the source can be operated up to $10.8 \mathrm{~W}(1.8 \mathrm{~A}, 6 \mathrm{~V})$ at which the active area reaches a maximum temperature of $1248 \mathrm{~K}$ in air. The source is coupled to an elliptical reflector (25.4 $\mathrm{mm}$ diameter) so that the majority of optical output is concentrated within an approx $8 \mathrm{~mm}$ circle, $12 \mathrm{~mm}$ from reflector. Using the black-body radiation formula corrected by the emissivity, integrating over a solid angle of $2 \pi \mathrm{sr}$, and between $1 \mu \mathrm{m}$ and $22 \mu \mathrm{m}$, we find that the IR-12K source needs to be heated up to $560 \mathrm{~K}$ to radiate $0.52 \mathrm{~W}$ as required for a $15 \mathrm{~K}$ difference across the silicon rod. From the source data sheet, this would correspond to feeding about an electrical power of $3 \mathrm{~W}$. A temperature increase of the source filament is expected due to our operation in vacuum, with respect to the in-air calibration produced by the manufacturer. In fact, by operating in vacuum, for a given current the source filament gets hotter and thus less electrical power is needed to achieve a given source temperature and thus a given temperature difference.

On top of the vacuum box top we placed side by side, 4 Peltier cells (20.9 W max power, 2.2 A max current) which we connected in series: on their top we placed a heat sink (thermal resistance $0.33^{\circ} \mathrm{C} / \mathrm{W}, 250 \mathrm{~mm} \times 200 \mathrm{~mm} \times 40 \mathrm{~mm}$ ). A cut on the top side of the Polystyrene box allows for the heat sink to emerge and thus exchange heat with the room. To increase further the heat exchange with the laboratory room, on the lab floor, close to the table, we had a fan circulating air on top of the sink. In such conditions with an (electrical) cooling power of $34 \mathrm{~W}$ to the Peltier cells, we lowered $T_{1}$ by $9 \mathrm{~K}$ with respect to the laboratory temperature: this corresponds to a gain of $0.26 \mathrm{~K} / \mathrm{W}$. The time scale to reach a steady-state is order of tens of hours. This is due to the large masses and thermal resistances involved in the system.

Actually, to get an idea of the critical components we developed a model with lumped elements of the apparatus following the well known analogy between the equa- 
tions governing heat flows and those for electrical current flows. According to the electrical analogy, temperatures are represented by voltages, and heat fluxes by currents. Each mechanical piece is represented as a combination of thermal resistors and capacitor as shown in fig. 4. The resistance represents the thermal impedance: we distinguish between conduction $R_{c}$ and radiative $R_{r}$ resistances, depending on the mechanism involved in the heat exchange. Thermal resistances are computed according to:

$$
R_{c}=\frac{L}{k A} \quad, \quad R_{r}=\frac{1}{2 \epsilon \sigma A T_{0}^{3}}
$$

where $L$ is the estimated shortest path heat can follow, $A$ the mass cross section with respect to the thermal path $L$, $k$ the thermal conductivity, $\epsilon$ the surface emissivity and $T_{0}$ the temperature of the radiant surface. Here we consider small temperature differences between radiative surfaces with respect to their temperature which we then equate to $T_{0}$. Heat capacities of mechanical pieces are represented by capacitors and are computed according to:

$$
C=\rho V c
$$

where $\rho$ is material density, $V$ is the mass volume and $c$ is the specific heat. Table 1 lists the thermal-mechanical properties of the materials used in the apparatus and table 2 lists the resistance and capacitance computed for each mechanical piece: time constants $R_{c} C$ of the order of 8-10 hours are characteristic of the vacuum chamber top and steel masses, which explains the above mentioned time scale to reach the steady state.

For the data acquisition and temperature control we used commercial PXI instrumentation. The PXI instru-

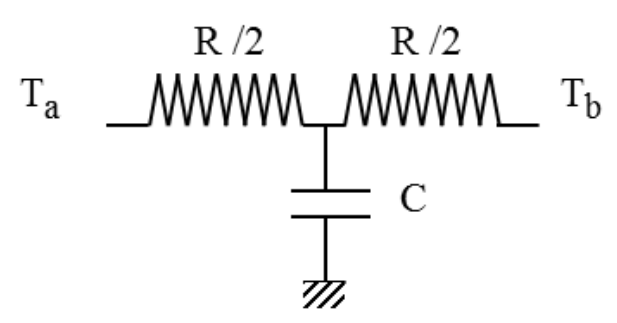

Fig. 4. Electrical lumped element circuit representing a mass subject to a temperature difference $T_{a}-T_{b}$. For $\mathrm{R}$ and $\mathrm{C}$ values see discussion in the text. In the electric model, $T_{a}$ and $T_{b}$ represent the voltages at the extreme of the circuit.

Table 1. Density $(\rho)$, thermal conductivity $(k)$ and specific heat $(c)$ of the materials employed in our apparatus.

\begin{tabular}{lccc}
\hline Material & $\rho\left[\frac{\mathrm{kg}}{\mathrm{m}^{3}}\right]$ & $k\left[\frac{\mathrm{W}}{\mathrm{mK}}\right]$ & $c\left[\frac{\mathrm{J}}{\mathrm{kgK}}\right]$ \\
\hline Steel AISI 304 & 7800 & 15 & 503 \\
Al alloys & 2760 & 130 & 915 \\
Polystyrene & 35 & 0.033 & 1400 \\
Copper & 8200 & 400 & 385 \\
Teflon & 2150 & 0.2453 & 1047 \\
Silicon & 2330 & 149 & 705 \\
\hline
\end{tabular}

mentation includes a programmable dc power supply which we use for powering the Peltier cells. 


\begin{tabular}{|l|r|r|}
\hline Part & $C\left[\frac{J}{K}\right]$ & $R_{c}\left[\frac{K}{W}\right]$ \\
\hline Springs (parallel of 3) & 50 & 5.2 \\
\hline Shape A load mass & 8700 & 1.4 \\
\hline Shape B load mass & 6900 & 4.5 \\
\hline Copper screws & 27 & 0.74 \\
\hline Flange & 2700 & 0.01 \\
\hline Oscillator - Support & 3690 & 0.00077 \\
\hline Oscillator - Beam & 0.001 & 29 \\
\hline Oscillator - End Mass & 200 & 0.15 \\
\hline Vacuum chamber - Top & 12500 & 2.5 \\
\hline Vacuum chamber - Tube & 8000 & 230 \\
\hline Vacuum chamber - Base & 21800 & 4.3 \\
\hline Polystyrene box (6 faces) & 2860 & 0.47 \\
\hline
\end{tabular}

Table 2. Heat capacities $C$ and conduction resistances $R_{c}$ of different mechanical parts. Part notation is as in fig. 1.

\section{Implementation of the thermal control}

A rough estimation of the feasibility of our cooling strategy and of required power can be achieved with a back-ofthe envelope calculation as follow: let us consider a silicon rod, $L=0.085 \mathrm{~m}$ in length, $5 \mathrm{~mm}$ diameter, that we want to subject to a temperature difference $\Delta T=15 \mathrm{~K}$. Then, as stated, we need to flow a power $W \sim 0.52 \mathrm{~W}$. Let the rod be attached to a cylindrical mass, $0.2 \mathrm{~m}$ in diameter (hence section $A=0.0314 \mathrm{~m}^{2}$ ) which is kept at a temperature $T_{0}=303 \mathrm{~K}$. Then we need to remove the power $\mathrm{W}$ from the cylindrical mass: if this is to be done radiatively (here we consider only the radiation exchanged by one of the flat surfaces of the cylindrical mass, meaning that the other flat surface is occupied by the oscillator and the curved surface is small), the surface which the mass is faced to needs to be at a temperature lower by at least: $W /\left(4 T_{0}^{3} A \sigma\right)=2.6 \mathrm{~K}$ where $\sigma$ is the Stefan-Boltzmann constant. With the above estimated gain, this would require an electric power to the cells of about $10 \mathrm{~W}$. These numbers seemed feasible thus we proceeded in implementing the temperature control.

To stabilize the oscillator base temperature $T_{1}$ we decided to implement a PID feedback control: the loop consists of the oscillator end mass, the NTC thermistor measuring $T_{1}$, the PXI data acquisition and signal processing unit, the programmable power supply and the Peltier cells. We developed a $C \#$ code in Visual Studio: the program samples simultaneously all thermometers, typically at a rate of $0.1 \mathrm{~Hz}$, computes the current to be fed to the cells according to a given algorithm, pilots the programmable power supply so that it gives the required power and appends thermometer readings in a file for postprocessing. The output signal out is updated at the same rate the thermometers are sampled. At the $i$-th iteration with $i=1,2 \ldots$, the implemented algorithm computes the following output signal:

$\operatorname{out}[i]=K \cdot \operatorname{err} T[i]+\frac{K}{\tau_{I}} \cdot I_{i}+K \cdot \tau_{D} \cdot \frac{(\operatorname{err} T[i]-\operatorname{err} T[i-1])}{t_{i}-t_{i-1}}$

where the integral $I_{i}$ is:

$$
I_{i}=\operatorname{err} T[i]\left(t_{i}-t_{i-1}\right)+I_{i-1}
$$

and $\operatorname{err} T[i]=T_{\text {set }}-T_{1}[i]$ is the error signal, $T_{1}[i]$ being the $i$-th reading of the thermistor measuring $T_{1}$ and $T_{\text {set }}$ being the set point for the control loop of $T_{1} \cdot \tau_{I}$ and $\tau_{D}$ are 
the parameters that scale the integral and derivative controller, respectively. We also implemented an antiwindup scheme in the algorithm to limit the integral control within a lower and an upper bound.

We used the Ziegler-Nichols closed-loop tuning method for determining the optimal PID parameters: we closed the loop with only the proportional control on and found the minimum proportional gain which causes sustained oscillation of $T_{1}$. This turned out to be $K_{u}=0.8 \mathrm{~A} / \mathrm{K}$ and the period of these oscillations is $P_{u}=90 \mathrm{ks}=25 \mathrm{~h}$. Thus the optimal PID parameter are: $K=0.6 \times K_{u}=0.48 \mathrm{~A} / \mathrm{K}$, $\tau_{I}=P_{u} / 2=45000 \mathrm{~s}$ and $\tau_{D}=P_{u} / 8=11250 \mathrm{~s}$. These are also the PID parameters that we used in the algorithm to control $T_{1}$.

We decided not to activate a feedback loop to control $T_{2}$ and hence the temperature difference across the oscillator. In fact no temperature disturbances are produced inside the vacuum box, apart the effect of the IR heater and of the power $(\sim 0.1 \mathrm{~W})$ consumed by the oscillator amplifier which we leave switched on: on the other hand, any change in the laboratory temperature would enter as a disturb into the system, firstly affecting the vacuum box and then propagating to the oscillator base and finally to the oscillator end mass. Thus our strategy is: to control $T_{1}$ for reducing the effect of the noise and fixing the working temperature of the oscillator and to feed a constant current to the heater for setting the temperature difference, which depends only on the thermal resistance of the rod. So the heater current sets the temperature difference while the system set-point (ie $T_{1}$ ) is determined by the interplay

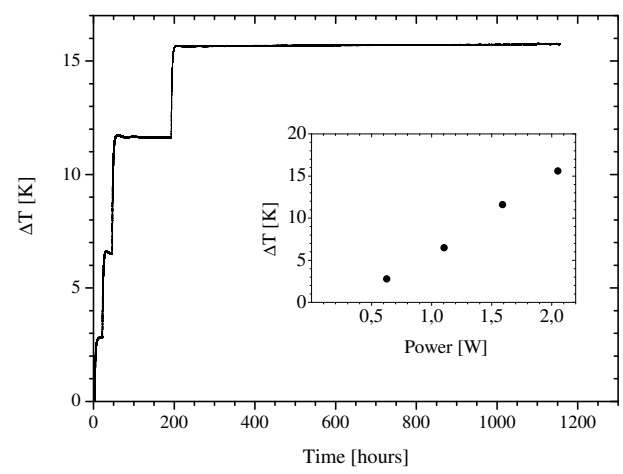

Fig. 5. Measurements of the temperature difference $\Delta T$ across the oscillator as function of time while $T_{1}$ was stabilized and while feeding constant power to the IR source in 4 steps. The inset shows the temperature difference as function of the electric power to the source.

of heater and Peltier actuators but depends mainly on the Peltier actuator.

\section{Experimental results}

We started an experimental run to test both the control of $T_{1}$ and the setting of the temperature difference $T_{2}-T_{1}$. The gas pressure inside the chamber (as measured at the pump head) never exceeded $10^{-5}$ mbar. We first stabilized the base temperature $T_{1}$ to $T_{\text {set }}=297.0 \mathrm{~K}$ and then fed constant power to the heater in 4 steps and measured the corresponding difference $\Delta T=T_{2}-T_{1}$. The data are summarized in fig. 5 .

Thus we are able to set temperature differences as high as $15 \mathrm{~K}$ which is within our requirements; the corresponding electrical power which we need to feed to the IR source 

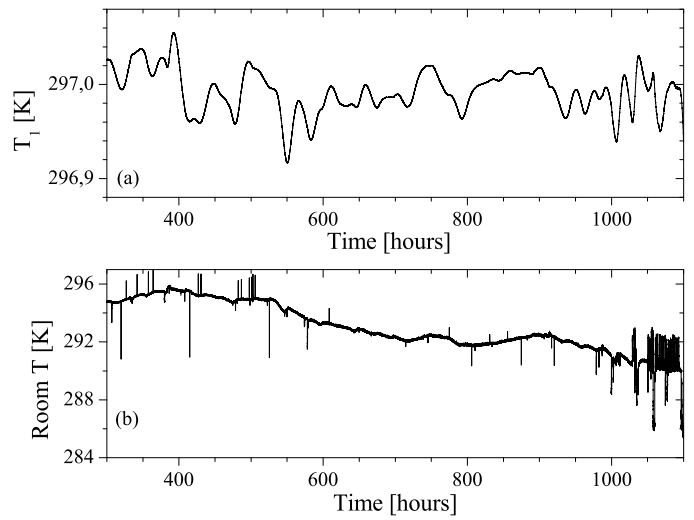

Fig. 6. (a) Behavior of $T_{1}$ while the PID control loop was active. (b) Behavior of laboratory temperature during the same period. The plotted time span corresponds to 33 days of continuous operation.

is about $2 \mathrm{~W}$, thus less than the predicted $3 \mathrm{~W}$ estimated in sec. 2.1, as anticipated.

Fig. 6 shows the measurements of $T_{1}$ and of the laboratory temperature data after the maximum temperature difference was set: the plotted time span is more than 1 month long. The data show that $T_{1}$ never exceeded from within a band of $0.138 \mathrm{~K}$ during the plotted 800 hours. In the meantime the laboratory temperature shown in fig. 6 - (b) changed by up to $10 \mathrm{~K}$ : thus the control loop is effective in reducing the temperature changes by a factor 70 as sensed at the oscillator base. We stress that these data were collected while feeding maximum power (namely $2.74 \mathrm{~V}, 0.749 \mathrm{~A}$ ) to the heater.

Fig. 7 is the histogram of the $T_{1}$ measurements shown in fig. 6 - (a). From it we compute that $72 \%$ of the $T_{1}$ measurements lay within the interval $(297.00 \pm 0.025) \mathrm{K}$ and almost $96 \%$ of the $T_{1}$ measurements lay within the interval (297.00 \pm 0.05$) \mathrm{K}$ during the 33 days period ana-

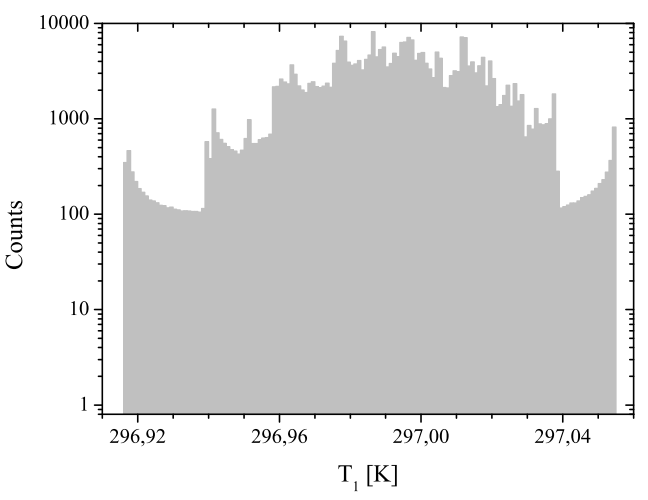

Fig. 7. Histogram of the $T_{1}$ measurements shown in fig. 6 - (a) and collected in 33 days.

lyzed: within this limit our control loop reduces by a factor 100 the changes of the room temperature.

Fig. 8 - (a) shows the measurements of $T_{2}$ performed using the thermopile (top, black curve: $T_{2, T}$ ) and the Pt100 (bottom curve: $T_{2, P t}$ ). The difference in the measurements is due to a systematic, calibration error: here and for all other thermometers we decided not to correct the calibration errors since they do not affect our results. It appears evident that the thermopile, which has in-built electronics, is much noisier than the Pt100 readings; a sudden voltage drop occurred on the $T_{2, T}$ data, which does not correspond to a real temperature change and is in fact absent form the $T_{2, P t}$ data. Fig. 8 - (b) shows the computed temperature difference $\Delta T=T_{2, P t}-T_{1}$ : the scatter of the measurements is due to the $T_{2, P t}$ data. A positive time derivative of $\Delta T$ is evident, in spite of a constant electric power to the heater: this is likely due to a slow change of heater performance in the vacuum environment. To the purpose of setting a constant temperature difference across the oscillator, this drift does not actually require an automatic 

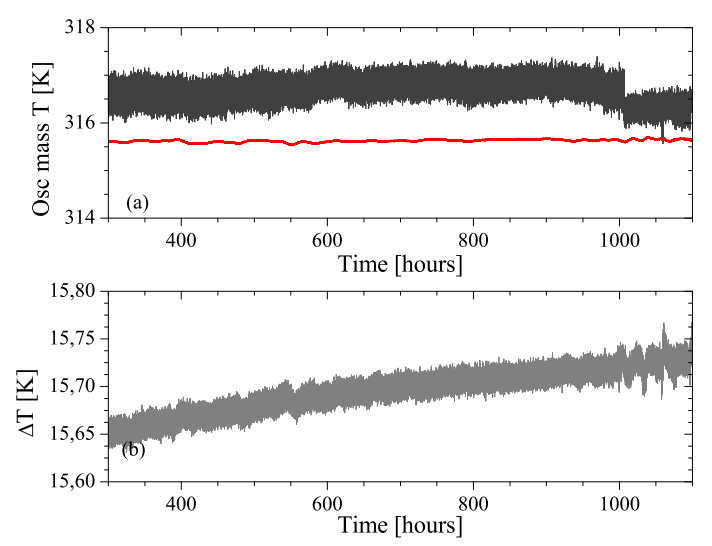

Fig. 8. Behavior of (a) the oscillating mass temperature as read by the thermopile (ie $T_{2, T}$, top curve) and by the Pt100 thermometer (ie $T_{2, P t}$, bottom curve) and (b) of the thermal difference $\Delta T=T_{2, P t}-T_{1}$ during the same 33 day long time span shown also in fig. 6 .

control loop but can be removed by a manual change of the electric power to the heater, once in a few days.

\section{Conclusions}

We have developed an experimental setup which can host a payload $\lesssim 6.6 \mathrm{~kg}$ in mass, up a few liters in volume, in a vacuum environment, with reduced mechanical noise and with an active thermal control. We made use of radiative heat transfer and non-contact thermometers to minimize interfering with the experimental apparatus. We quantify the thermal control performance by comparing the room temperature variations with those of the controlled quantity over a period of about 1 month. We evidenced that in the long term we can reduce the former by a factor about 100 , even in presence of an internal, dc heat source of $2 \mathrm{~W}$.

\section{Acknowledgements}

The research leading to these results has received funding from the European Research Council under the European Community's Seventh Framework Programme (FP7/20072013) / ERC grant agreement n 202680. The EC is not liable for any use that can be made on the information contained herein.

\section{References}

1. See for instance: G. K. McMillan and C. M. Toarmina, Advanced Temperature Measurement and Control (ISA: The Instrumentation, Systems, and Automation Society, 1995)

2. See for instance: D. J. Mead, Passive Vibration Control (Wiley, New York 1999)

3. L. Conti, M. Bonaldi and L. Rondoni, Class. Quantum Grav. 27, 084032 (2010)

4. M. Saraceni et al., Rev. Sci. Instrum. 81, 035115 (2010)

5. M. J. Persky, Rev. Sci. Instrum. 70, 2193 (1999)

6. I. Mellouki, O. Touayar, T. Ktari, N. Yacoubi, Infrared Physics \& Technology 45, 273 (2004)

7. I. Mellouki, N. Bennaji, N. Yacoubi, Infrared Physics \& Technology 50, 58 (2007) 